\title{
Giant Prolactinomas: Outcomes of Multimodal Treatments for 42 Cases with Long-Term Follow-Up
}

Authors

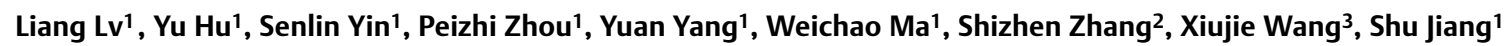

\section{Affiliations}

1 Department of Neurosurgery, Pituitary Adenoma Multidisciplinary Center, West China Hospital of Sichuan University, China

2 Department of Neurosurgery, The Second Affiliated Hospital of Guangzhou Medical University, Guangzhou, China

3 Laboratory of Experimental Oncology, West China Hospital, West China Clinical Medical School, Sichuan University, Chengdu, China

Key words giant prolactinoma, prolactin, dopamine agonist, bromocriptine

received 26.01.2018

revised $\quad 15.03 .2018$

accepted 24.03.2018

\section{Bibliography}

DOI https://doi.org/10.1055/a-0597-8877

Published online: 25.6.2018

Exp Clin Endocrinol Diabetes 2019; 127: 295-302

(C) . A. Barth Verlag in Georg Thieme Verlag KG Stuttgart .

New York

ISSN 0947-7349

\section{Correspondence}

Shu jiang

West China Hospital of Sichuan University

No. 37, Guoxue Alley

Chengdu, 610041

China

Tel.: + 86/28/62539 221, Fax: + 86/28/62539 221

jiangshu2000@126.com

Xiujie Wang

Laboratory of Experimental Oncology

West China Hospital

West China Clinical Medical School

Sichuan University

No. 1 Keyuan Road 4
Gaopeng Avenue

High-Tech Zone, Chengdu

Sichuan 610041

P.R. China

Tel.: + 86/28/685164 018, Fax: + 86/28/85164018

xiujiewang@sina.com, xiujiewang@scu.edu.cn

\section{ABSTRACT}

Giant prolactinomas represent a rare entity of pituitary tumors so that the management of these patients is still a prevalent challenge at present. Paying special attention to the treatment strategy and outcomes, we presented a large series of 42 cases looking forward to share our understanding and experience in management of these patients. Male patients accounted for $71.4 \%$ of this series and were relatively younger $(35.70 \pm 2.42$ vs. $52.00 \pm 3.55$ years, $p=0.0011$ ) and harbored bigger tumors (14.57 vs. $7.74 \mathrm{~cm}^{3}, \mathrm{p}=0.0179$ ) compared to females. Almost all of these tumors showed suprasellar extension (97.6\%) and cavernous sinus invasion (92.9\%). Dopamine agonist represented an efficient method to control PRL concentrations (98.8\%) and reduce tumor burdens (81.2\%). PRL normalization was detected in 13 out of the 27 patients initially treated with bromocriptine (BRC) whereas none of the 14 patients with firstline operation gained a normalization of PRL concentration after surgery. Although there was no reliable predictor of tumor response, First PRL reduction was a predictive criterion for the nadir PRL level during the long-time period of follow-up for first-line bromocriptine treatment. In conclusion, patients with giant prolactinomas did not gain more benefits from initial surgery. Dopamine agonist (BRC) should be first-line treatment for giant prolactinomas whereas operation merely served as a remedy for acute compression symptoms and dopamine agonist resistance. Consecutive monitoring of serum PRL levels in the early stage of initial BRC treatment is useful for evaluation of therapeutic effect and further therapeutic decision. 


\section{Introduction}

Prolactinomas, or prolactin (PRL)-secreting pituitary adenomas, represent the most common type of pituitary adenomas with the estimated incidence ranging from 2.2 to 77.5 per 100000 people [1-3]. Microprolactinomas constitute the most predominant portion of prolactinomas. However, giant prolactinomas, which are defined as adenomas with large size and extremely high serum PRL concentrations, are relatively rare entity [4]. It was reported that giant prolactinomas only accounted for $0.5 \%$ of all pituitary adenomas and were found predominantly in men [5-6].

Actually, there is no consensus definition of giant prolactinoma. From the point of tumor diameter, adenoma with more than $4 \mathrm{~cm}$ in diameter is usually regarded as giant adenoma. But clinical diagnosis of giant prolactinoma requires additional evidences. In nonsurgical treated patient, owing to the absence of pathological confirmation of diagnosis, it is reasonable to include a high serum PRL level in the diagnosis criteria of giant prolactinoma. Accordingly, several cutoff points of serum PRL level had been proposed and applied in clinical management and research, but a PRL level>1000 ng/ $\mathrm{mL}$ was recommended in the most recent studies [7-9].

Generally, the therapeutic goals for prolactinomas include normalization of PRL and reduction of tumor size without causing pituitary dysfunction. However, owing to the size and invasive growth pattern of giant prolactinomas, the optimum treatment strategies are not well established yet. Dopamine agonists, including bromocriptine (BRC) and the preferred cabergoline ( $C A B)$, are still the first-line treatment option for their high efficiency in both reductions of PRL level and tumor size [10]. Surgery serves as another choice mainly for patients intolerant or resistant to dopamine agonists. Besides, surgery are also recommended as initial therapy to those patients exhibited pituitary apoplexy with severe clinical symptoms or acute intracranial hypertension or patients who bear massive extrasellar extended adenomas with high risk of visual impairment $[11,12]$. For refractory cases, radiotherapy, especially gamma knife radiosurgery, and temozolomide are also considered $[13,14]$. Consecutive monitoring of serum PRL concentration and tumor size are two crucial aspects in management of giant prolactinomas. After multimodal treatment, significant decrease of tumor size (86-93.8\% of tumor volume) led to obvious improvement of compression symptoms $[4,15,16]$. However, the reported PRL normalization rate was still at a relatively low level (37.5-61\%), and there was no reliable predictor for therapeutic outcomes in the literature [16-19].

Herein, we retrospectively reported a large cohort of patients with giant prolactinomas in our institution. Focusing on the outcomes of different therapeutic interventions, we analyzed the clinical characteristics expecting to provide a better understanding of management strategy for giant prolactinoma.

\section{Patients and Methods}

\section{Patients' selection}

We retrospectively collected the medical archives of patients registered in our Pituitary Adenoma Multidisciplinary Center for giant prolactinoma in West China Hospital of Sichuan University between January 2009 and December 2015. The diagnosis criteria for giant prolactinoma were summarized as follows: a pituitary adenoma with a maximum diameter of (or more than) $4 \mathrm{~cm}$ in any direction; or an extremely high level of serum PRL concentration (>1000 ng/ $\mathrm{mL}$ ) which was verified by standard assay; co-secretion of growth hormone $(\mathrm{GH})$ or adrenocorticotropic hormone (ACTH) was excluded [4]. Therefore, according to all these criteria strictly, a total of 42 patients were enrolled in this study.

\section{Neuroradiological evaluation}

MR images were acquired in each patient using standard 3.0-T scanner with and without contrast enhancement at diagnosis. Followup MRIs were acquired annually thereafter. Besides, for operated patients, additional MR scanning was also performed within $72 \mathrm{~h}$ and 3 months after surgery to evaluate the degree of surgical resection. Suprasellar tumor growth was considered significant in those cases with obvious compression of the optic chiasm [16]. Cavernous sinus invasion was considered in tumors extended beyond a line through the cross-sectional centers of the intracavernous and supracavernous internal carotid arteries (ICAs). Sphenoid sinus or clivus/ petrous apex extension was verified if the lesion grew into the sphenoid sinus or apparently invaded clivus/petrous apex [12, 16]. In addition, tumor volume was verified using the Region of Interest (ROI) function of MRI system of our institution and calculated as the sum of all tumor slabs measured on each tumor slice multiplied by slice thickness [20]. Additionally, cystic components were included in tumor volume only in evaluation of tumor size reduction.

\section{Endocrinological study}

Serum PRL levels were measured by commercial electro-chemiluminescence kits (ROCHE Diagnostics, IN, USA). The intra-assay coefficients of variation (CVs) for PRL concentrations of 23.5 and $238.67 \mathrm{ng} / \mathrm{mL}$ were 1.16 and $0.95 \%$, respectively. The sensitivity was $0.065 \mathrm{ng} / \mathrm{mL}$. The analytic measurement range (AMR) was $0.105-452.88 \mathrm{ng} / \mathrm{mL}$. The reference range was $4.6-21.4 \mathrm{ng} / \mathrm{mL}$ for males and 6.0-29.9 ng/mL for females. More importantly, in all patients, serum samples were diluted ( 100 -fold) before determination allowing for the 'hook effect' [4, 21]. Other hormones, including cortisol, ACTH, free tetraiodothyronine (free T4), free triiodothyronine (free T3), thyroid stimulating hormone (TSH), luteinizing hormone (LH), follicle-stimulating hormone (FSH), estradiol, testosterone, insulin-like growth factor 1 (IGF-1) and $\mathrm{GH}$, were measured through commercially available kits (ROCHE Diagnostics, IN, USA). The First PRL reduction (\%) was defined as the percentage of PRL level decrease at the first month after oral dopamine agonist administration as contrasted with basal PRL level. Besides, nadir PRL level was the lowest value in the follow-up duration after intervention. Moreover, PRL index was defined as serum PRL concentration dividing by tumor volume.

\section{Pathological study}

For operated patients, surgical specimens were verified by routine hematoxylin \& eosin staining and further immunohistochemistry (IHC) for GH, PRL, ACTH, TSH, LH and FSH by our colleagues of department of pathology. 
- Table 1 General Characteristics of total population.

\begin{tabular}{|c|c|c|c|c|}
\hline Variables ${ }^{1}$ & Total & Female & Male & P value \\
\hline No. of patients & 42 & 12 & 30 & \\
\hline Age at diagnosis (years, mean \pm SD) & $40.36 \pm 2.42$ & $52.00 \pm 3.55$ & $35.70 \pm 2.42$ & 0.0011 \\
\hline Delay of diagnosis [years, median (IQR)] & $2.5(3.0)$ & $9.0(6.5)$ & $2.0(2.5)$ & 0.0003 \\
\hline Median volume ${ }^{2}\left(\mathrm{~cm}^{3}, \mathrm{IQR}\right)$ & $14.02(8.01)$ & $7.74(8.45)$ & $14.57(5.93)$ & 0.0179 \\
\hline Median maximum diameter ${ }^{2}(\mathrm{~cm}, \mathrm{IQR})$ & $4.10(0.24)$ & $4.15(0.37)$ & $4.10(0.29)$ & 0.7358 \\
\hline Median basal PRL (ng/mL, IQR) & $5551(7555)$ & $3776(5103)$ & $5873(8424)$ & 0.2703 \\
\hline Median PRL index $\left[\mathrm{ng} /\left(\mathrm{mL} \cdot \mathrm{cm}^{3}\right), \mathrm{IQR}\right]$ & $511.70(419.46)$ & $574.64(109.60)$ & $418.01(530.88)$ & 0.5470 \\
\hline Cystic component (yes/no) & $18 / 24$ & $5 / 7$ & $14 / 16$ & 1.0000 \\
\hline \multicolumn{5}{|l|}{ Extending region (No. of patients) } \\
\hline Suprasellar cistern/ Third ventricle & 41 & 12 & 29 & 0.1265 \\
\hline Sphenoidal sinus & 28 & 6 & 22 & \\
\hline Cavernous sinus & 39 & 12 & 27 & \\
\hline Clivus/ Petrous apex & 9 & 0 & 9 & \\
\hline Median nadir PRL (ng/mL, IQR) & $19.96(112.72)$ & $22.02(106.50)$ & $18.60(92.71)$ & 0.7502 \\
\hline Median latest PRL (ng/mL, IQR) & $28.61(193.05)$ & $141.05(192.19)$ & $24.74(283.45)$ & 0.7439 \\
\hline PRL normalization (No. of patients) & 21 & 5 & 16 & 0.6776 \\
\hline Median PRL reduction (\%, IQR) & $98.8(3.5)$ & $96.8(4.9)$ & $99.1(1.7)$ & 0.1918 \\
\hline Median tumor shrinkage (\%, IQR) & $79.0(18.9)$ & $82.0(18.6)$ & $76.5(18.0)$ & 0.5291 \\
\hline \multicolumn{5}{|c|}{ 1. Data were displayed in the form of mean \pm standard deviation (SD) or median (interquartile range, IQR) for quantitative data; } \\
\hline \multicolumn{5}{|c|}{ 2. Cystic components were subtracted from the measurement of tumor volume and tumor maximal diameter; } \\
\hline \multicolumn{5}{|c|}{ SD: standard deviation; IQR: interquartile range } \\
\hline
\end{tabular}

\section{Statistical analysis}

Results were displayed in the form of mean \pm standard deviation (SD) for normal distributed data or median for skewed distributed data. Student's T and Mann-Whitney tests were used for quantitative data whereas Chi-square or Fisher's exact tests were performed for comparison of categorical data. Spearman rank correlations were employed for correlations between basal PRL level and tumor volume or between First PRL reduction (\%) and nadir PRL level. Linear regression analysis was conducted for tumor volume and basal PRL level after logarithmic transformation whereas non-linear regression analysis was employed for PRL Reduction (\%) and nadir PRL level after outlier elimination. And binary logistic regression was employed for analyzing predictive variables of PRL normalization. A p value of $<0.05$ in two-sided tests was considered significant. Data were analyzed using SPSS version 17.0 (SPSS Chicago IL, USA).

\section{Results}

\section{Patients' characteristics}

A total of 42 patients were enrolled in this retrospective analysis ( $\triangleright$ Table 1). A male predominance (male: female $=5: 2$ ) has been identified in this cohort. The overall mean age at diagnosis was $40.36 \pm 2.42$ years (range, $17-67$ years) and male patients were relatively younger than females $(35.70 \pm 2.42$ vs. $52.00 \pm 3.55$ years, $\mathrm{p}=0.0011)$. The delay in diagnosis was also shorter in males than females (2.0 vs. 9.0years, $p=0.0003$ ).

Initial complaints included intermittent headaches in 23 patients, visual impairment in 36 cases (including 3 patients with di- plopia), amenorrhea in 9 females but obvious galactorrhea in 6 women. Besides, six male adults initially complained about erectile dysfunction or decreased libido and a pubertal patient (17 years) suffered from hypoplasia of male gonads. Another patient registered in our institution for repeated epistaxis. Moreover, an incidentaloma was diagnosed in an asymptomatic male.

\section{Tumor characteristics}

At presentation, all of these patients suffered from prominent hyperprolactinemia with median serum PRL concentration being $5551 \mathrm{ng} / \mathrm{mL}$ ( Table 1). The median initial tumor volume was $14.02 \mathrm{~cm}^{3}$. Significantly, male patients had bigger tumors than females ( 14.57 vs. $7.74 \mathrm{~cm}^{3}, \mathrm{p}=0.0179$ ). However, there was no difference in PRL index between female and male patients (574.64 vs. $\left.418.01 \mathrm{ng} /\left(\mathrm{mL} \cdot \mathrm{cm}^{3}\right), \mathrm{p}=0.5470\right)$. Thus, it seemed that there was no correlation between tumor volume and basal PRL level. Indeed, basal PRL concentrations had no correlation with tumor size in males (Spearman rank correlation coefficient, $r_{s}=0.2982$, $p=0.2210)$ or females $\left(r_{s}=0.4855, p=0.1146\right)$, separately. However, when enrolling all cases, it turned to be a significant outcome $\left(r_{s}=0.4266, p=0.0048\right.$, $>$ Fig. 1$)$.

Moreover, giant prolactinomas caused significant compression or invasion to surrounding structures ( $\triangleright$ Table 1 ). The vast majority of lesions presented suprasellar cistern/ third ventricle extension (41 cases) or cavernous sinus invasion (39 cases). Sphenoidal sinus invasion was verified in 28 cases (including one case of nasopharynx invasion). Clivus/ petrous apex erosion was detected in 9 males. In addition, cystic prolactinomas accounted for $42.9 \%$ (18 cases) of this series. In addition, all these tumor characteristics of invasiveness were consistent in two genders. 


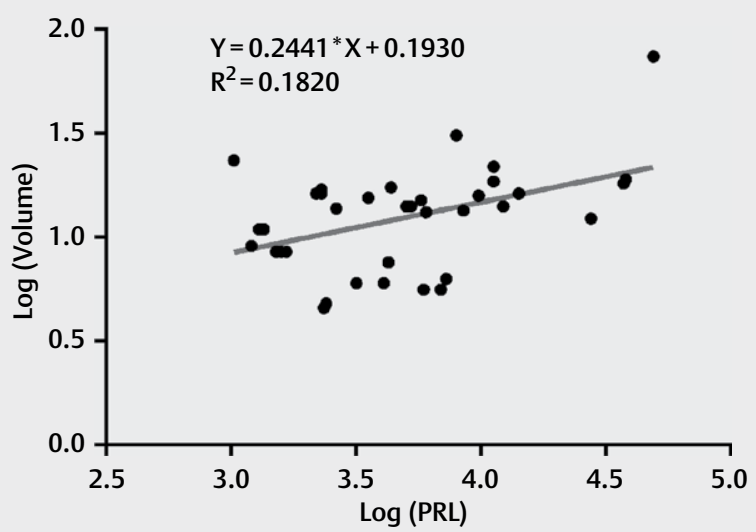

-Fig. 1 Correlation of basal PRL and tumor volume. Spearman rank correlation coefficient $\left(r_{s}\right)$ was $0.4266(p=0.0048)$. And further linear regression analysis revealed a quantitative relation of $\log (\mathrm{PRL})$ and Log(Volume) $\left(r^{2}=0.1820, p<0.0001\right)$.

\section{Treatment strategies and outcomes}

Dopamine agonist served as first-line treatment in 27 patients (64.3\%). Unfortunately, BRC is the exclusive kind of dopamine agonist which is available in mainland of China. Thus, all of these patients were treated with BRC at a initial dosage of $7.5 \mathrm{mg} /$ day whether as first-line, second-line or third-line treatment ( $>$ Fig. 2). Gastrointestinal reaction (nausea and vomiting) and postural hypotension presented in only 2 and 1 patients of the whole case series, respectively. Fortunately, both of these side effects of BRC were overcome through gradual dose escalation. PRL normalizaion was detected in 13 out of the 27 patients initially treated with BRC. And based on latest MRI scanning, tumor volume decreased by $80.3 \%$. However, 2 females showed partial response to BRC and were operated thereafter. Before operations (transsphenoidal microscopic approach), the PRL levels were as high as 2188 and $956.6 \mathrm{ng} / \mathrm{mL}$ with a relative high dosage of BRC (15.0 mg/day), respectively. Moreover, MRIs revealed no obvious tumor shrinkage in both patients after BRC treatment. Gamma knife radiosurgery were adopted as adjuvant treatment for postoperative residual for both females. However, no PRL normalization was verified untill their last visit.

Operation served as first-line treatment in 14 patients. Transcranial approach was employed in 4 cases whereas adenomas in remaining 10 cases were resected by transsphenoidal microscopic approach. Four patients received operations for acute pituitary apoplexy which led to obviously worse visual field and severe hypopituitarism. Although dopamine agonist and operation were equally recommended as an initial therapeutic option allowing for the obvious extrasellar extension, 9 patients selected operation to prevent further visual field impairment based on their own preference. Moreover, the remaining one patient chose transsphenoidal surgery based on his individual strong desire despite that dopamine agonist was strongly recommended. As a consequence, the mean degree of resection was $69.2 \pm 6.7 \%$ (range, $24.7 \sim 95.2 \%$ ) based on postoperative MRI evaluation. Accordingly, there was no PRL normalization being detected in these operated patients, and BRC treatment was adopted as second-line therapy thereafter. In addition, tumor regrowth presented in 2 cases with a relatively short period of time ( 3 and 5 months after surgery, respectively) and they received a second transsphenoidal microscopic operation. Another 2 patients were re-operated for intratumoral hemorrhage of remanents via transsphenoidal microscopic approach. There was no severe surgical complication, like cerebrospinal fluid rhinorrhea, visual loss, intracranial infection or internal carotid artery rupture, presenting in operated cases. Besides, two patients received postoperative gamma knife radiosurgery.At the time of the latest follow-up, the median tumor shrinkage was 79.7\%. PRL normalization was verified in 8 out of 14 patients after subsequent BRC treatment.

Additionally, one male patient received $B R C$ as initial treatment after gamma knife radiosurgery in another hospital. But partial response to BRC (PRL level was $4981 \mathrm{ng} / \mathrm{mL}$ at a dosage of $22.5 \mathrm{mg} /$ day without tumor size reduction) led to a transsphenoidal adenoidectomy for him. However, resistance to BRC persisted after surgery, which was partly improved by combination of BRC and temozolomide (PRL level declined to 781.1 from $4444 \mathrm{ng} / \mathrm{mL}$ ).

After a mean follow-up duration of $60.0 \pm 4.6$ months (range, 12-96 months), the median PRL level was $28.61 \mathrm{ng} / \mathrm{mL}$ at the last visit. But the nadir PRL level $(19.96 \mathrm{ng} / \mathrm{mL})$ seemed to be lower in the follow-up duration. As a result, based on the latest endocrine study, only 21 patients ( $50.0 \%$ ) obtained PRL normalization with a median PRL reduction of $98.8 \%$. As another therapeutic goal, significant tumor shrinkage was observed in this cohort. The median tumor shrinkage was $81.2 \%$ after multimodal intervention. Additionally, complete tumor regression was detected through MRI in 3 patients and two of them discontinued BRC treatment but failed to be persistent endocrine remission. Obviously, with respect to first-line treatment, pure BRC treatment contributed to more satisfactory outcomes than operation alone (PRL normalization rate, $48.1 \%$ vs. $0 \%, p=0.0017)$. Nevertheless, there was no statistic difference between BRC treatment alone and combination of BRC and operation ( $48.1 \%$ vs. $57.1 \%, p=0.5464$ ).

Additionally, parameters, like age at diagnosis, gender, delay of diagnosis, basal PRL level and tumor volume, were not predictive factors for endocrine remission assessed based on latest PRL concentrations or nadir PRL levels in binary logistic regression model (data not shown). Howerver, further analysis revealed that PRL reduction at the first month after BRC treatment (First PRL reduction) significantly correlated with nadir PRL level $\left(r_{s}=-0.8397\right.$, $\mathrm{p}<0.0001$, Fig. 3 ) in 27 patients who were initially treated with BRC $(7.5 \mathrm{mg} /$ day). Additionally, the median First PRL reduction was $96.7 \%$ (range, 40.9-99.8\%).

\section{Visual field and pituitary function}

Visual field deficit (36 cases) was one of the most common symptoms in this series ( $\triangleright$ Table 2 ). The majority (28 cases) of these cases manifested as typical bitemporal hemianopsia. Unilateral blindness was presented in 7 patients and the remaining one showed homonymous hemianopsia. Satisfactorily, none of the operated patients suffered from deterioration of visual field after surgery. Besides, the vast majority of patients (83.3\%) acquired improvement of visual field. In detail, partial and apperant improvement was achieved in 19 and 11 cases, respectively. 


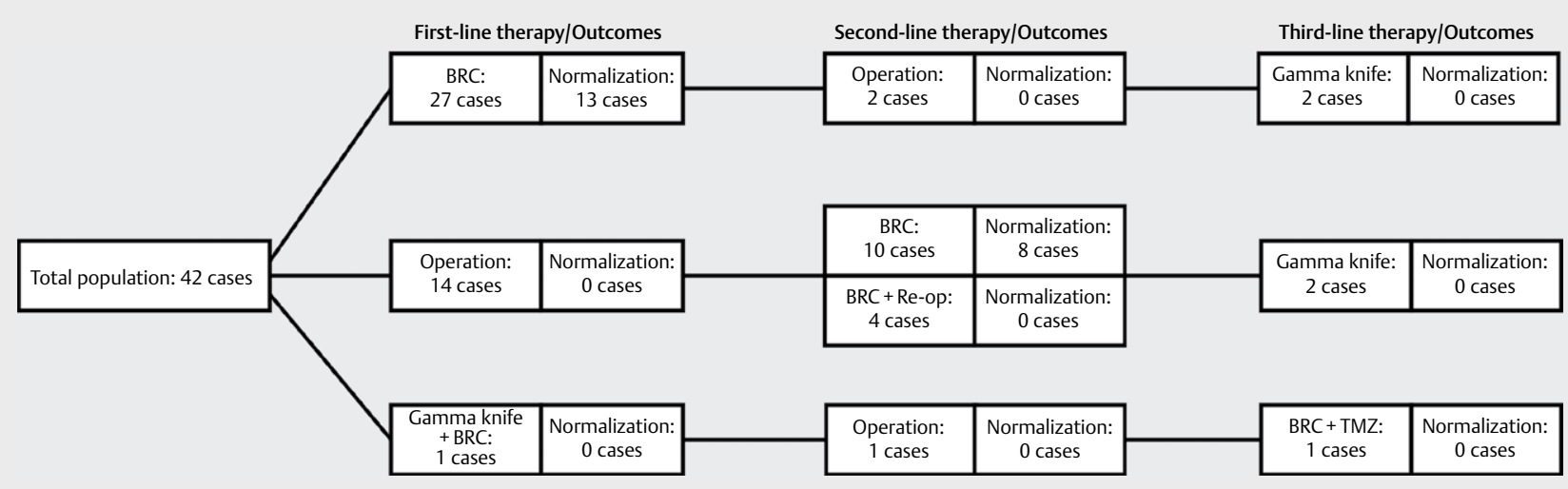

- Fig. 2 Therapuetic options and outcomes flow diagram. BRC: bromocriptine; Re-op: re-operation; TMZ: temozolomide.

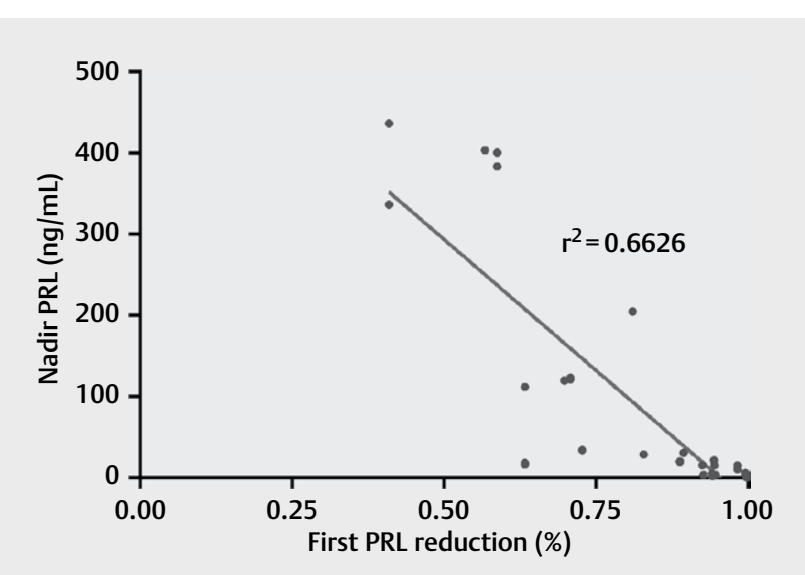

- Fig. 3 Correlation of First PRL reduction and nadir PRL. Spearman rank correlation coefficient $\left(r_{s}\right)$ was $-0.8397(p<0.0001)$. And further non-linear regression analysis revealed a quantitative relation after elimination of outliers $\left(r^{2}=0.6626, p<0.0001\right)$.

Male central hypogonadism (50.0\%) and central hypothyroidism $(66.7 \%)$ were the most common abnormalities of pituitary function before treatment. GH deficiency, female central hypogonadism and central hypocortisolism accounted for 15, 9 and 5 cases, respectively. After initial surgery, newly-diagnosed male central hypogonadism, central hypothyroidism and central hypocortisolism were presented in 3, 2 and 2 patients, respectively. However, no apparent improvement of pituitary function was identified immediately after surgery. At the last visit, only 9 patients persistently suffered from central hypothyroidism whereas low testosterone level and low estradiol was detected in 15 males and 6 females, respectively. $\mathrm{GH}$ deficiency and central hypocortisolism were verified only in 5 and 2 patients.

In addition, compared to BRC treatment, patients did not gain more benefit from surgery. There was no statistical difference in visual field improvement and pituitary function recovery between two groups of patients.

\section{Discussion}

In current study, we reported the clinical characteristics and therapeutic outcomes of a large cohort of giant prolactinomas. Dopamine agonist (BRC) was an efficient method to control PRL concentration and reduce tumor burden. For patients initially treated with $B R C$, the First PRL reduction predicted the nadir PRL level during the period of follow-up. Surgical cure of giant prolactinomas could not be regarded as a rational therapeutic target. Relief of acute intrasellar hypertension caused by pituitary apoplexy or compressive symptoms that failed to be settled by DAs were two of the main targets of surgical intervention.

Prolactinoma, especially microadenoma, is prevalent tumor in females [22]. However, there is an apparent male predominance for giant prolactinoma with a male to female ratio of 9:1 [4]. In present study, male patients accounted for $71.4 \%$ of 42 cases and were relatively younger than females. Moreover, male patients also harbored bigger tumors. In several previous studies, this sex-related difference in prolactinoma size was attributed to the relatively long time of the asymptomatic phase in males $[23,24]$. However, the greater growth potential of tumor in males might also contribute to this result [25].

The relationship of tumor size and PRL level had been discordantly reported in macro- and giant prolactinomas $[5,26]$. Compared to non-giant prolactinomas, this relationship of PRL level and tumor size was usually weaker in giant prolactinomas [27]. Underestimation of PRL level due to "hook effect" and hemorrhagic or cystic components within tumors might be two of the crucial factors that led to this discordance between tumor size and hormonal level. In our study, all serum PRL levels were determined after 100fold dilution and cystic components were also subtracted from the total tumor volume. Although in a relatively low degree, there was a positive correlation between tumor size and PRL level among the whole case series.

Regarding therapy, DAs should be served as the first-line therapy for prolactinomas. Patients with giant prolactinomas also show a fairly good response to DAs with large reductions in serum PRL level and tumor burden. However, the relative large size and extreme hyperprolactinemia often hinder the PRL normalization. At present, cabergoline $(C A B)$ is the preferred dopamine agonist in 
- Table 2 Visual field and pituitary function.

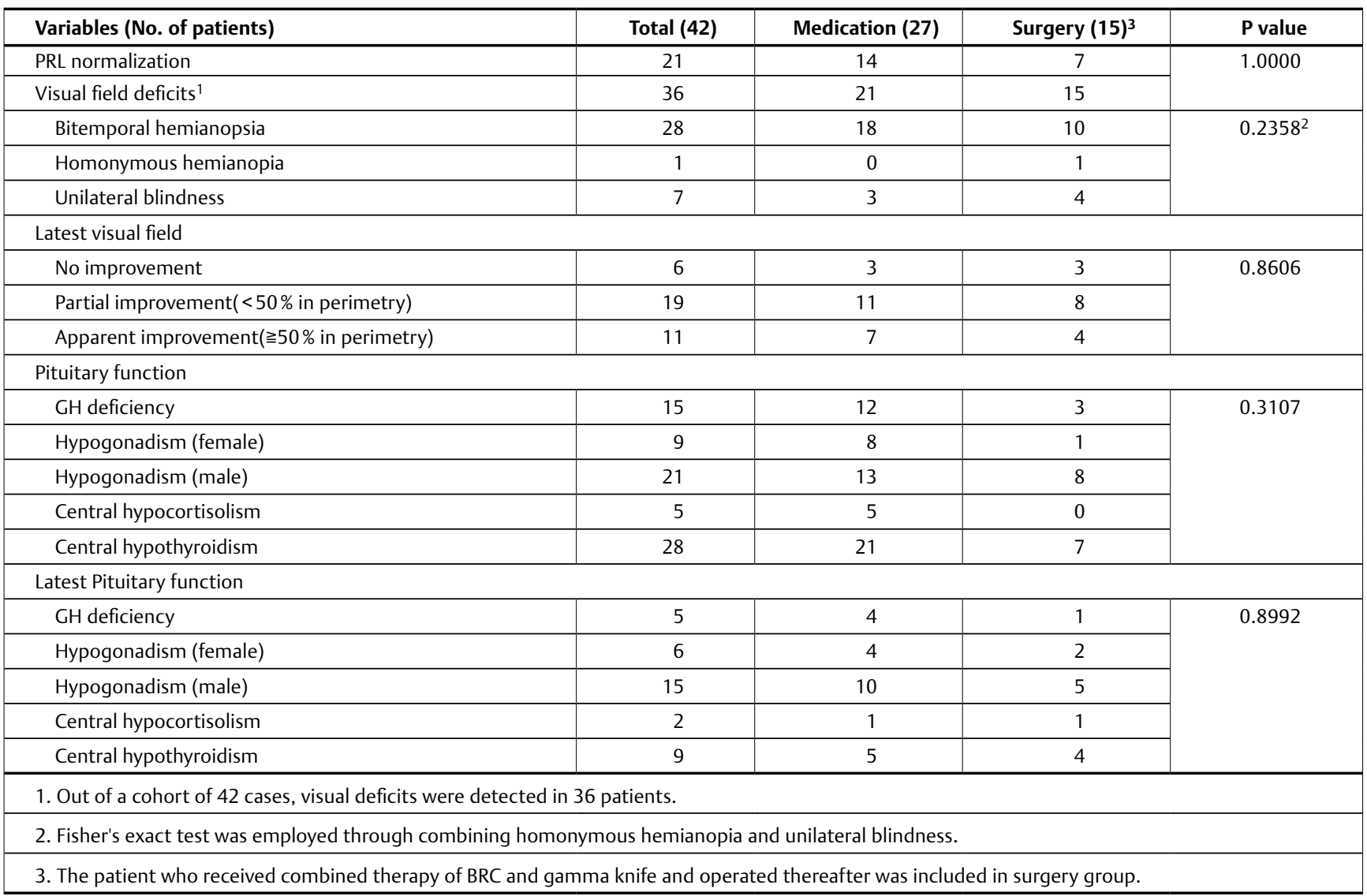

management of prolactinomas. However, bromocriptine (BRC) also presents equally efficiency in giant prolactinomas when compared with CAB [4]. In the current study, BRC first-line treatment was employed in 27 patients and resulted in a PRL normalization rate of $48.1 \%$. Operations show relatively limited value for prolactinomas, especially giant prolactinomas. Surgery is usually reserved for patients who are resistant to or unable to tolerate the required dose of DAs. Acute compression symptoms are also indications of surgical treatment. Nevertheless, surgery rarely resulted in normalization of PRL concentration [4]. The invasiveness has always been a major obstacle for surgical removal of pituitary adenomas. Unfortunately, consistent with previous studies, all of the lesions in this study invaded surrounding structures, including suprasellar cistern/ third ventricle (97.6\%), sphenoidal sinus (66.7\%), cavernous sinus $(92.9 \%)$ and clivus/ petrous apex $(21.4 \%)[6,16,28]$. Thus, operations resulted in a resection degree of approximate $70 \%$ in 14 patients who were initially treated with surgery, and no total resection or PRL normalization was verified after surgery. Besides, it was reported that tumor debulking might be followed by better response to DAs treatment [15] whereas the three patients who showed partial response to BRC in this series did not obtain better outcomes of BRC treatment after surgery. Allowing for the relatively low surgical mortality and incidence of surgical complications nowadays [9], operation is reserved as an available option. Nevertheless, it must be mentioned that surgical cure of giant prolactinomas could not be regarded as a rational therapeutic target. Op- erations should not be recommended even for adenomas with apparent suprasellar extension before DA treatment failure. Relief of acute intrasellar hypertension caused by pituitary apoplexy or compressive symptoms that failed to be settled by DAs are the main targets of surgical intervention.

After long-term follow-up, PRL concentration decreased by more than $95 \%$ and tumor size also decreased by $75 \%$. However, only a half of the cases achieved normalization of PRL concentration at the last visit. In fact, a part of endocrine controlled patients underwent a process of reduction of drug dose (even discontinuity in 2 cases). Therefore, re-increasing of PRL level might be one of the reasons contributed to the low endocrine remission rate (50.0\%) [12]. Although tumor remnants presented in 39 out of 42 patients, most of the remnants were well tolerated and more than $80 \%$ of these cases showed visual field improvement after multimodal therapy. Allowing for the relatively low PRL normalization rate, relief of compressive symptoms through tumor size reduction could be regarded as a pragmatic and short-term target for giant prolactinomas.

Just as previously reported, there was no reliable indicator of tumor response in the whole cohort of 42 cases $[17,29]$. However, for the 27 patients who initially treated with BRC ( $7.5 \mathrm{mg} /$ day), First $P R L$ reduction presented significant predictive ability for nadir PRL level. Many authors have reported that dramatic decrease of PRL concentration and improvement of symptoms could be observed within several days after BRC or CAB treatment [30-32]. So, for 
cases that are sensitive to dopamine agonists, the therapeutic effects may emerge in the very early stage of treatments. Thus, our results indicated that First PRL reduction might be a sensitive predictor of tumor response to BRC intervention and be useful for further therapeutic decision in management of giant prolactinomas.

Visual field deficits and endocrine disorders are manifestations that remarkably impact on the quality of life in patients. Fortunately, regardless of therapeutic approaches, most of the patients experienced improvement in visual fields and pituitary functions. In general, visual field deficits are caused by compression to the optic tracts or chiasma. Thus, decompression, via either medication or surgery, could provide the possibility for visual field improvement. In our cohort of patients, BRC presented the same effect as surgical intervention from the aspect of visual field improvement. Therefore, visual field deficits alone should not be regarded as a surgical indication for giant prolactinomas. In other words, DAs should also be recommended to patients with compressive symptoms for that $85.7 \%$ of cases presented visual improvement by BRC alone. Hypopituitarism usually caused by direct destruction or compression of the pituitary gland by local invasive tumors or compression of the portal vessels and pituitary stalk caused by pituitary apoplexy [33]. Besides, hyperprolactinemia is also responsible for low gonadal hormones level [25]. Consistent with previous studies [6, 16, 26], after multimodal treatments, the recovery of central hypothyroidism had been detected in a considerable amount of patients $(67.9 \%)$ with replacement treatment. However, there were still a half of patients suffered from central hypogonadism at the last visit. Persistent hypogonadism could be overcome with hormones replacement. Nevertheless, it was reported that estradiol replacement has potential risk of stimulating both PRL synthesis and release as well as lactotroph cell proliferation [34]. On the contrary, another study demonstrated that there was no evidence of tumor enlargement in hypogondal women with hormones replacement [35]. More importantly, exogenous androgen impairs the function of innate hypothalamic-pituitary-gonadal axis, although gonadal hormones replacement could improve the clinical symptoms. So, in our institution, stimulatory therapy with or without gonadal hormones replacement was merely recommended for patients with strong desire of fertility. Thus, the relatively high incidence of hypogonadism after medical intervention in our study could partially attribute to the therapeutic strategy for hypogonadotropic hypogonadism and a relatively low natural recovery rate of $50 \%$ that was reported in a recent review [12].

\section{Conclusion}

In conclusion, BRC presented high efficiency in both PRL reduction and tumor shrinkage. Patients with giant prolactinomas did not gain more benefits from surgery. DA (BRC) should be first-line treatment for giant prolactinomas whereas operation was merely served as a remedy for acute compression symptoms and DA resistance. Consecutive monitoring of serum PRL levels in the early stage of initial treatment was useful for evaluation of therapeutic effects and further treatment strategy.

Ethical approval: The materials used in this study consisted only of archival specimens from this department. All procedures followed ethical standards of the Declaration of Helsinki 1975, as revised in 1983 and approved by the Institutional Review Board of our institute.

\section{Acknowledgements}

The authors thank financial support from funding (Grant No. 2016SZ0015) owned by Dr. Feng Ye.

\section{Funding}

This research was financially supported by Foundation of Science and Technology Department of Sichuan province (Grant No. 2015SZ0120 and Grant No. 2016SZ0015).

\section{Conflict of Interest}

The authors report no conflict of interest concerning the materials or methods used in this study or the findings specified in this paper.

\section{References}

[1] Daly AF, Rixhon M, Adam C et al. High prevalence of pituitary adenomas: A cross-sectional study in the province of Liege, Belgium. The Journal of Clinical Endocrinology and Metabolism 2006; 91: 4769

[2] Raappana A, Koivukangas ], Ebeling T et al. Incidence of pituitary adenomas in Northern Finland in 1992-2007. The Journal of Clinical Endocrinology and Metabolism 2010; 95: 4268-4275

[3] Colao A, Savastano S. Medical treatment of prolactinomas. Nature Reviews Endocrinology 2011; 7: 267-278 doi:10.1038/nrendo.2011.37

[4] Maiter D, Delgrange E. Therapy of endocrine disease: The challenges in managing giant prolactinomas. European journal of endocrinology / European Federation of Endocrine Societies 2014; 170: R213-R227 doi:10.1530/EJE-14-0013

[5] Espinosa E, Sosa E, Mendoza V et al. Giant prolactinomas: Are they really different from ordinary macroprolactinomas? Endocrine 2016; 52: 652-659 doi:10.1007/s12020-015-0791-7

[6] Corsello SM, Ubertini G, Altomare M et al. Giant prolactinomas in men: Efficacy of cabergoline treatment. Clinical Endocrinology 2003; 58 : 662-670

[7] Shrivastava RK, Arginteanu MS, King WA et al. Giant prolactinomas: Clinical management and long-term follow up. Journal of Neurosurgery 2002; 97: 299

[8] Cho EH, Lee SA, Chung JY et al. Efficacy and safety of cabergoline as first line treatment for invasive giant prolactinoma. Journal of Korean Medical Science 2009; 24: 874-878 doi:10.3346/jkms.2009.24.5.874

[9] Gillam MP, Molitch ME, Lombardi G et al. Advances in the treatment of prolactinomas. Endocrine Reviews 2006; 27: 485-534 doi:10.1210/ er.2005-9998

[10] Dash S, Annamalai AK, Simpson HL et al. Acute shrinkage of a giant prolactinoma, masquerading as an erosive skull base tumour, with cabergoline. QJM 2013; 106: 85

[11] Besser M. Criteria for medical as opposed to surgical treatment of prolactinomas. Acta Endocrinologica 1993; 129: (Suppl 1): 27

[12] Moraes AB, Silva CM, Vieira Neto L et al. Giant prolactinomas: The therapeutic approach. Clin Endocrinol (Oxf) 2013; 79: 447-456 doi:10.1111/cen.12242 
[13] Shrivastava RK, Arginteanu MS, King WA et al. Giant prolactinomas: Clinical management and long-term follow up. Journal of Neurosurgery 2002; 97: 299-306

[14] Corsello SM, Ubertini G, Altomare M et al. Giant prolactinomas in men: Efficacy of cabergoline treatment. Clinical Endocrinology 2003; 58: 662-670

[15] Shimon I, Sosa E, Mendoza $V$ et al. Giant prolactinomas larger than 60 $\mathrm{mm}$ in size: A cohort of massive and aggressive prolactin-secreting pituitary adenomas. Pituitary 2016; 19: 429-436 doi:10.1007/s11102016-0723-4

[16] Andujar-Plata P, Villar-Taibo R, Ballesteros-Pomar MD et al. Long-term outcome of multimodal therapy for giant prolactinomas. Endocrine 2017; 55: 231-238 doi:10.1007/s12020-016-1129-9

[17] Delgrange E, Daems T, Verhelst ] et al. Characterization of resistance to the prolactin-lowering effects of cabergoline in macroprolactinomas: A study in 122 patients. European Journal of Endocrinology 2009; 160: 747-752

[18] Yarman S, Kurtulmus N, Bilge A. Optimal effective doses of cabergoline and bromocriptine and valvular leasions in men with prolactinomas. Neuro Endocrinology Letters 2012; 33: 340

[19] Bevan JS, Webster J, Burke CW et al. Dopamine agonists and pituitary tumor shrinkage. Endocrine reviews 1992; 13: 220-240

[20] Hsu CY, Guo WY, Chien CP et al. MIB-1 labeling index correlated with magnetic resonance imaging detected tumor volume doubling time in pituitary adenoma. European journal of endocrinology / European Federation of Endocrine Societies 2010; 162: 1027-1033 doi:10.1530/ EJE-09-1100

[21] Comtois R, Robert F, Hardy J. Immunoradiometric assays may miss high prolactin levels. Annals of Internal Medicine 1993; 119: 173

[22] Ciccarelli A, Daly AF, Beckers A. The epidemiology of prolactinomas. Pituitary 2005; 8: 3

[23] Leonard MP, Nickel C], Morales A. Hyperprolactinemia and impotence: Why, when and how to investigate. Journal of Urology 1989; 142: 992-994

[24] Guay AT, Sabharwal P, Varma S et al. Delayed diagnosis of psychological erectile dysfunction because of the presence of macroprolactinemia. The Journal of Clinical Endocrinology and Metabolism 1996; 81: 2512
[25] Delgrange E, Trouillas ], Maiter D et al. Sex-related difference in the growth of prolactinomas: A clinical and proliferation marker study. The Journal of clinical endocrinology and metabolism 1997; 82: 21022107

[26] Chattopadhyay A, Bhansali A, Masoodi SR. Long-term efficacy of bromocriptine in macroprolactinomas and giant prolactinomas in men. Pituitary 2005; 8: 147-154 doi:10.1007/s11102-005-5111-4

[27] Gillam MP, Molitch ME, Lombardi G et al. Advances in the treatment of prolactinomas. Endocrine reviews 2006; 27: 485

[28] Shimon I, Benbassat C, Hadani M. Effectiveness of long-term cabergoline treatment for giant prolactinoma: Study of 12 men. European journal of endocrinology / European Federation of Endocrine Societies 2007; 156: 225-231 doi:10.1530/EJE-06-0646

[29] Yang MS, Hong JW, Lee SK et al. Clinical management and outcome of 36 invasive prolactinomas treated with dopamine agonist. Journal of neuro-oncology 2011; 104: 195-204

[30] Iglesias P, Macho LP, Díez J]. Resolution of macroprolactinoma-induced symptomatic hydrocephalus following cabergoline therapy. Age Ageing 2004; 33: 410-412

[31] Cackett P, Eunson G, Bath L et al. Proptosis as the presenting sign of giant prolactinoma in a prepubertal boy: Successful resolution of hydrocephalus by use of medical therapy. Future Oncology 2012; 8: 1621-1626

[32] Perani D, Colombo N, Scotti G et al. Rapid size reduction of giant prolactinoma following medical treatment. Journal of Computer Assisted Tomography 1984; 8: 131

[33] Arafah BM. Reversible hypopituitarism in patients with large nonfunctioning pituitary adenomas. The Journal of Clinical Endocrinology and Metabolism 1986; 62: 1173

[34] Gillam MP, Middler S, Freed D] et al. The novel use of very high doses of cabergoline and a combination of testosterone and an aromatase inhibitor in the treatment of a giant prolactinoma. The Journal of clinical endocrinology and metabolism 2002; 87: 4447-4451 doi:10.1210/jc.2002-020426

[35] Molitch ME. Medical treatment of prolactinomas. Endocrinology and Metabolism Clinics of North America 1999; 28: 143-169 